\title{
Decomposition behavior of III/V semiconductor precursor gases in a closed gas cell in-situ TEM holder observed by mass spectrometry
}

\author{
Maximilian Widemann ${ }^{1}$, David Krug${ }^{2}$, Felix Gruber ${ }^{2}$, Andreas Beyer ${ }^{3}$ and Kerstin Volz \\ ${ }^{1}$ Materials Science Centre and Department of Physics, Philipps University Marburg, Hans-Meerwein- \\ Straße 6, Marburg, 35032, Germany, Marburg, Hessen, United States, ${ }^{2}$ Materials Science Centre and \\ Department of Physics, Philipps University Marburg, Hans-Meerwein-Straße 6, Marburg, 35032, \\ Germany, United States, ${ }^{3}$ Materials Science Centre and Department of Physics, Philipps University \\ Marburg, Hans-Meerwein-Straße 6, Marburg, 35032, Germany, Germany
}

III/V semiconductor devices are used for many technological applications, e.g. LEDs, lasers and solar cells. A widely used fabrication process for these materials is metal organic vapor phase epitaxy (MOVPE), where metal organic precursor gases are supplied to a heated substrate. The precursor gases decompose thermally and the growth material adsorbs onto the crystalline substrate resulting in a layer by layer crystal growth. Mass spectrometry analysis of the gas phase in the MOVPE reactor is able to give detailed insights into the decomposition of the precursor gases, and thus into the growth process [1]. Insitu studies of the growth process promise an improvement of the performance of the fabricated materials, however direct observations of the crystal growth in conventional MOVPE reactors are challenging. Insitu (scanning) transmission electron microscopy ((S)TEM) allows the investigation of dynamic processes, which occur during growth of III/V semiconductors. Gas environmental cell and heating holders enable to supply gases while heating the sample so that conditions comparable to those during the MOVPE process can be realized in any microscope [2] and semiconductor growth can be performed. The comparability of such a micro reactor with a conventional one, however, still needs to be proven.

To this end, a commercially available Protochips in-situ system, equipped with a quadrupole mass spectrometer working with $70 \mathrm{eV}$ electron ionization, has been modified to investigate the processes occurring during semiconductor growth. In order to allow the usage of toxic and pyrophoric gases, like the precursor gases used in MOVPE growth, a gas mixing system, an appropriate gas monitoring system as well as a gas scrubbing system have been added to the setup [3]. A double CS-corrected JEOL JEM 2200FS operating at $200 \mathrm{kV}$ was used for the TEM observations. Investigated precursor gases are tertiarybutylphosphine (TBP) and trimethylgallium (TMGa), which are used for gallium phosphide growth at partial pressures between 10-1 and 10-3 hPa and a V/III ratio of around 10. Additionally, N2 is used as carrier gas at pressures in the range of $102 \mathrm{hPa}$. Temperatures at which efficient decomposition takes place are expected to be above $450{ }^{\circ} \mathrm{C}$ [4].

We observe the thermal decomposition behavior of the precursor gases in the micrometer scaled TEM holder tip as well as the influence of the irradiating electron beam interacting with the gas volume.

Figure 1 and 2 show mass spectra at $750{ }^{\circ} \mathrm{C}$ and $900{ }^{\circ} \mathrm{C}$ of the thermal decomposition species of TBP. The spectra were recorded with a gas mixture of $99 \%$ nitrogen and 1\% TBP with a total pressure of 100 $\mathrm{hPa}$. They were background corrected and the contribution of the nitrogen and undecomposed TBP molecule signal was subtracted. Additionally, they were normalized to the phosphine peak intensity at 34 $\mathrm{u}$ and plotted to the same scale. In this way, only the signal of TBP decomposition species are visible and a comparison of their ratio is feasible. The experiment was performed without an impinging electron beam to see only the effect of temperature. Both spectra show intensities indicating the presence of phosphine $(31 \mathrm{u}$ to $34 \mathrm{u})$, and a changing ratio of isobutane and isobutene with varying temperature. Increasing the temperature from $750{ }^{\circ} \mathrm{C}$ to $900{ }^{\circ} \mathrm{C}$ especially reduces the intensity at $43 \mathrm{u}$, the base peak 
position of isobutane, whereas the $39 \mathrm{u}, 41 \mathrm{u}$ and $56 \mathrm{u}$ peaks, pertaining to isobutene, increase. Additionally, these findings give insight in the decomposition mechanisms. Isobutane is produced due to homolytic fission, whereas isobutene by beta-hydrogen elimination of TBP. The results show that the beta-hydrogen elimination becomes more favorable as decomposition mechanism for TBP with increasing temperature.

We demonstrate our findings on the thermal and electron beam induced decomposition behavior of III/V semiconductor precursor gases inside an in-situ closed gas cell TEM holder.

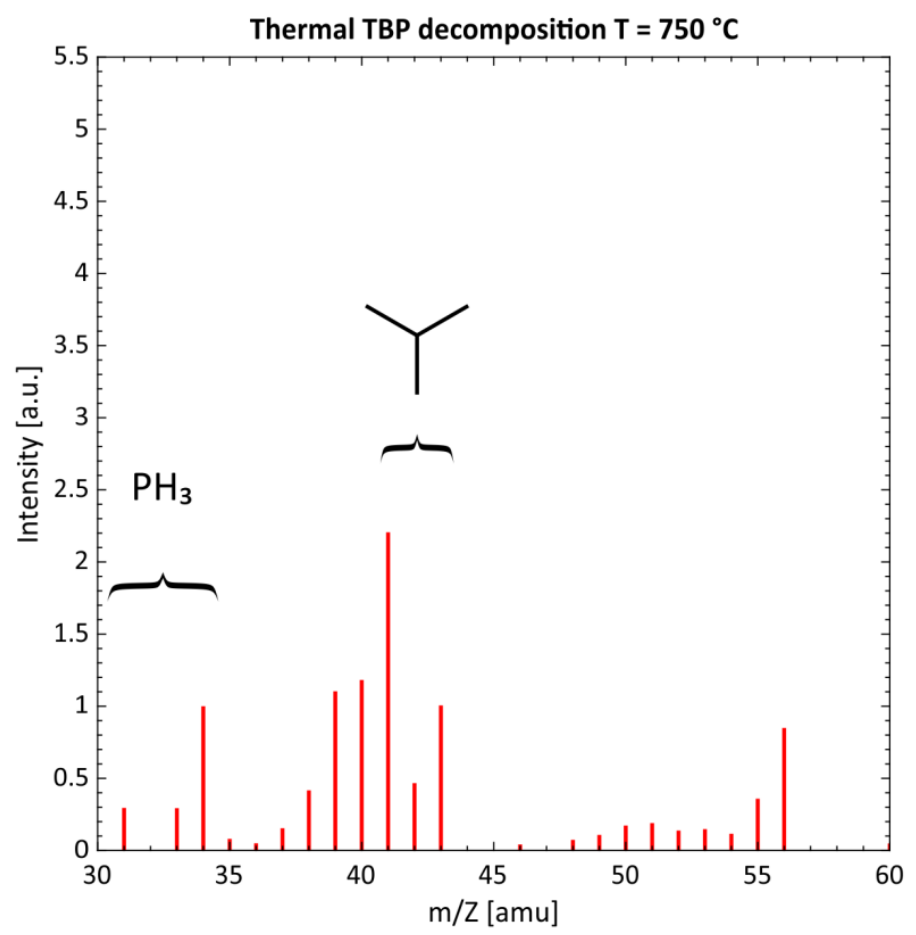

Figure 1. Mass spectrum of TBP decomposition species at $750{ }^{\circ} \mathrm{C}$ corrected with respect to the pure TBP and nitrogen intensities. The most dominant peak positions for phosphine and isobutane are indicated. 


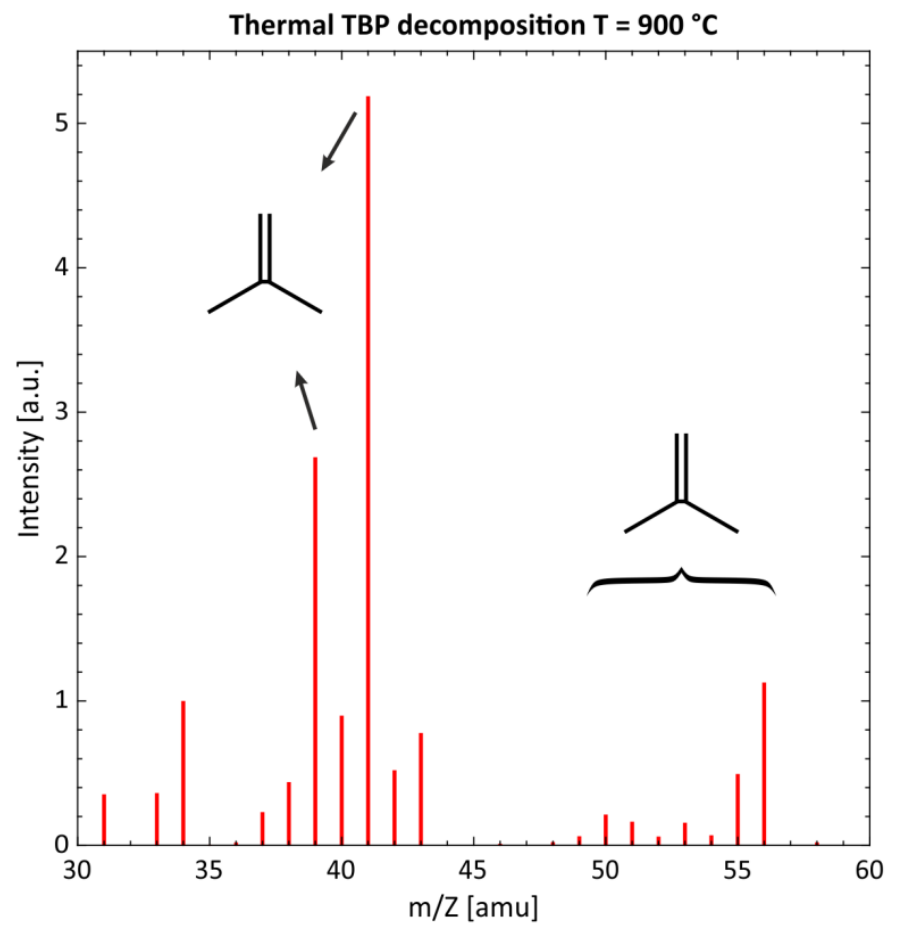

Figure 2. Mass spectrum of TBP decomposition species at $900{ }^{\circ} \mathrm{C}$ corrected with respect to the pure TBP and nitrogen intensities. Peak positions of isobutene peaks are indicated. Compared to the $750{ }^{\circ} \mathrm{C}$ spectrum the intensities of isobutane are decreased, whereas the one of isobutene are increased.

\section{References}

[1] P. W. Lee, et al., Journal of Crystal Growth 85.1-2 (1987), pp. 165-174.

[2] L. F. Allard, et al., Microscopy and Microanalysis 18.4 (2012), pp. 656-666.

[3] R. Straubinger, et al., Microscopy and Microanalysis 23.4 (2017), pp. 751-757.

[4] Li, S. H., et al., Journal of electronic materials 18.3 (1989), pp. 457-464. 